\title{
Dual-energy iterative reconstruction for material characterisation
}

\author{
B. Recur, M. Paziresh, G. Myers, A. Kingston, S. Latham and A. Sheppard \\ Australian National University, Dept Applied Maths, RSPE, Canberra, Australia
}

\begin{abstract}
In this paper, we develop a dual-energy ordered subsets convex method for transmission tomography based on material matching with a material dictionary. This reconstruction includes a constrained update forcing material characteristics of reconstructed atomic number $(Z)$ and density $(\rho)$ volumes to follow a distribution according to the material database provided. We also propose a probabilistic classification technique in order to manage this material distribution. The overall process produces a chemically segmented volume data and outperforms sequential labelling computed after tomographic reconstruction.
\end{abstract}

Keywords: Dual-Energy, X-Ray Tomography, Material Characterisation, Iterative Reconstructions

\section{INTRODUCTION}

In the field of material characterisation, dual-energy tomography can be used to recover the distribution of density, $\rho$, and atomic number, $Z$ (or average atomic number $Z_{\text {eff }}$ ), of a specimen by combining data acquired at two energies $E_{1}$ and $E_{2}$. Such a characterisation is based on an attenuation $\mu_{E}(x)$ model at each point $x$ of the acquired sample depending on both $\rho$ and $Z$ according to the acquisition energy $E:^{1}$

$$
\begin{aligned}
\mu_{E}(x) & =\frac{K_{1}}{E^{k}} Z^{n}(x) \rho(x)+K_{2} f_{K N}(E) \rho(x) \\
& =f_{e^{-}}^{E}(Z(x), \rho(x))+f_{C S}^{E}(\rho(x))
\end{aligned}
$$

where $K 1$ and $K 2$ are two constants. First part of Eq. (1) refers to the photoelectric effect, denoted $f_{e^{-}}^{E}(\cdot, \cdot)$, appearing for low energy $E$ and becoming negligible for high energies. The second part refers to the Compton scattering $f_{C S}^{E}(\cdot)$, depending on the Klein-Nishina formula $f_{K N}(E)$ defined by:

$$
f_{K N}(E)=\frac{1+\alpha}{\alpha^{2}}\left[\frac{2(1+\alpha)}{1+2 \alpha}-\frac{1}{\alpha} \ln (1+2 \alpha)\right]+\frac{1}{2 \alpha} \ln (1+2 \alpha)-\frac{(1+3 \alpha)}{(1+2 \alpha)^{2}} \text { where } \alpha=\frac{E}{510.975} \mathrm{keV}
$$

The first proposed algorithm ${ }^{1}$ consists of computing $\left[Z^{n} \rho\right]$-projections and $\rho$-projections from the both acquisitions to reconstruct $\left[Z^{n} \rho\right]$ and $\rho$ volumes directly using a standard tomographic algorithm. Since this approach does not take beam-hardening into account, a more comprehensive technique has been proposed ${ }^{2,3}$ to estimate $\left[Z^{n} \rho\right](x)$ and $\rho(x)$ volumes by a 2 -step process i) reconstructing the attenuation volumes $\mu\left(x, E_{1}\right)$ and $\mu\left(x, E_{2}\right)$, and ii) recovering material characteristic volumes from attenuation volumes by solving an equation system based on Eq. 1 for each energy. $Z(x)$ is often provided since it defines more accurately the chemical elements composing the acquired sample. However, extracting $Z(x)$ from $\left[Z^{n} \rho\right](x)$ is subject to the noise in $\rho(x)$, leading to errors in material characterisation.

In this paper, we propose a dual-energy iterative reconstruction method based on the ordered subsets convex (OSC) algorithm for transmission tomography using the attenuation function Eq. (1) directly in the forward model. During reconstruction, we estimate the specimen physical characteristics by matching each voxel with a material dictionary. This estimation is based on a probabilistic classification allowing us to allocate the most likely material in the dictionary for each voxel. The classification is verified during subsequent iterations of the algorithm, allowing false matchings to be corrected. The algorithm results in a reconstruction which is already segmented according to the physical properties of the scanned object.

Further author information (Send correspondence to B. Recur): benoit.recur@gmail.com

Developments in X-Ray Tomography IX, edited by Stuart R. Stock, Proc. of SPIE Vol. 9212,

$921213 \cdot$ (c) 2014 SPIE · CCC code: 0277-786X/14/\$18 · doi: 10.1117/12.2062442 


\begin{tabular}{|l|l|r|r|r|}
\hline Name & Chemical Formula & $\rho(\mathrm{g} / \mathrm{cc})$ & $Z_{\text {eff }}$ & \\
\hline Air & $\mathrm{Air}$ & 0.001 & 7.2240 & \\
\hline Iron Pure & $\mathrm{Fe}$ & 5.600 & 26.0000 & \\
\hline Rutile & $\mathrm{TiO}_{2}$ & 4.200 & 19.0006 & \\
\hline Siderite & $\mathrm{FeCO}_{3}$ & 3.960 & 21.0932 & \\
\hline Na-Montmorillonite & $\mathrm{NaAl}_{5} \mathrm{MgSi}_{12} \mathrm{O}_{30}(\mathrm{OH})_{6}$ & 2.650 & 11.4620 & \\
\hline Water & $\mathrm{H}_{2} \mathrm{O}$ & 1.000 & 7.5195 & \\
\hline Graphite & $\mathrm{C}$ & 2.300 & 6.0000 & \\
\hline Calcite & $\mathrm{CaCO}_{3}$ & 2.710 & 15.7100 & \\
\hline Dolomite & $\left.\mathrm{CaMg}_{2} \mathrm{CO}_{3}\right)_{2}$ & 2.870 & 13.7438 & \\
\hline Quartz Mineral & $\mathrm{SiO}_{2}$ & 2.650 & 11.5800 & \\
\hline Aluminium Pure & $\mathrm{Al}$ & 2.700 & 13.0000 & \\
\hline Anhydrite & $\mathrm{CaSO}_{4}$ & 2.950 & 15.6847 & \\
\hline Fused Quartz & $\mathrm{SiO}_{2}$ & 2.200 & 11.7842 & \\
\hline Pyrite & $\mathrm{FeS}_{2}$ & 5.020 & 21.9588 & \\
\hline Barite & $\mathrm{BaSO}_{4}$ & 4.500 & 47.2008 & \\
\hline Na-Feldspar & $\mathrm{NaAlSi}_{3} \mathrm{O}_{8}$ & 2.610 & 11.5534 & \\
\hline K-Feldspar & $\mathrm{KAlSi}_{3} \mathrm{O}_{8}$ & 2.530 & 13.3895 & \\
\hline Kaolinite & $\mathrm{Al}_{2} \mathrm{Si}_{2} \mathrm{O}_{5}(\mathrm{OH})_{4}$ & 2.600 & 11.1622 & \\
\hline Illite & $\mathrm{KAl}_{3} \mathrm{Si}_{3} \mathrm{O}_{10}(\mathrm{OH})_{2}$ & 2.800 & 9.6058 & \\
\hline Ca-Montmorillonite & $\mathrm{Ca}_{0.5} \mathrm{Al}_{5} \mathrm{MgSi}_{12} \mathrm{O}_{30}(\mathrm{OH})_{6}$ & 2.650 & 11.8277 & \\
\hline Chlorite & $\mathrm{Fe}_{2} \mathrm{Mg}_{2} \mathrm{Al}_{2} \mathrm{Si}_{2} \mathrm{~A}_{12} \mathrm{O}_{10}(\mathrm{OH})_{8}$ & 2.900 & 11.6449 & \\
\hline Celestite & $\mathrm{SrSO}_{4}$ & 3.900 & 30.4686 & \\
\hline Talc & $\mathrm{Mg}_{3} \mathrm{Si}_{4} \mathrm{O}_{10}(\mathrm{OH})_{2}$ & 2.750 & 8.4538 & \\
\hline Halite & $\mathrm{NaCl}^{2}$ & 2.350 & 15.3295 & \\
\hline
\end{tabular}

Table 1 . Density $\rho$ and average atomic number $Z_{\text {eff }}$ values for common materials. Colors correspond to the classification labels used in simulated/reconstructed volumes.

This iterative process avoids the independence of the successive steps in the existing sequence of segmentation and labelling. Indeed approximations during reconstruction can act dramatically on the rest of the sequence. Conversely iterative processing can correct for the labelling errors during the subsequent iterations. Additionally, several acquisition optimizations and dynamic tomography methods are developed by our group for the high coneangle helical-scanning micro-CT facility developed at ANU. These methods, proposed in companion papers, ${ }^{4,5}$ are based on iterative schemes which could ultimately include material characterisation in addition to their own processing aim.

Thus the remainder of the paper is organized as follows: In section 3 we investigate several material characterisation schemes based on numerical simulation. Our aim here is to define the most likely functions performing an efficient and noise-robust voxel material labelling with respect to $Z(x)$ and $\rho(x)$ volumes; or $\left[Z^{n} \rho\right](x)$ and $\rho(x)$ volumes. Then we introduce in section 4 the dual-energy iterative reconstruction algorithm including material characterisation during the iterations.

\section{MATERIAL AND METHODS}

\subsection{Material dictionary and numerical simulation}

This study is based on a numerical analysis of a synthetic object simulating a rock sample. This sample is modelled using the material database provided on Tab. 1. The numerical sample is composed of a cylindrical container filled randomly with different spherical mineral grains. The mineral composition we have chosen is $60 \%$ of quartz, $20 \%$ kaolinite and $20 \%$ calcite. The analytic object is rasterized into an $N^{3}$ voxel volume for comparison with reconstructed data (i.e. $N^{3}$ corresponds to reconstructed volume size). Fig. 1 shows the 2D central slices of $Z(x)$ and $\rho(x)$ volumes and a 3D multi-planar rendering coloured with the labels given in Tab. 1.

The synthetic object is acquired by a simulated acquisition using a detector 4 times with the number of pixels to prevent the inverse crime. ${ }^{6} \mathrm{X}$-ray lines (from source to each pixel of the detector) are traced with respect to the scanner geometry. Distances crossed by each X-ray line in each material composing the object are measured and total absorption is computed for each sub-pixel $i i$ by $A(i i)=\sum_{m \in M_{s}} \int \mu_{E, m}(x) d x$, where $M_{s}$ is the material set composing the sample and $\mu_{E, m}$ corresponds to $\mu_{E}$ (Eq. 1) for material $m$. We obtain the final projection value on the detector by averaging the four sub-pixel values and applying Poisson noise model:

$$
R(i)=\text { Poisson }\left\{\sum_{i i \in i} \gamma_{0} \exp (-A(i i))\right\}
$$

where $\gamma_{0}$ is the average photon counts on detector (blank scan) and $i i \in i$ denotes sub-pixel indexes contained in the detector pixel $i$. For example, Fig. 2 shows is a projection computed at $60 \mathrm{keV}$ and $120 \mathrm{keV}$ from the 


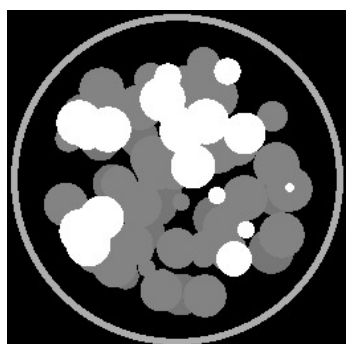

(a)

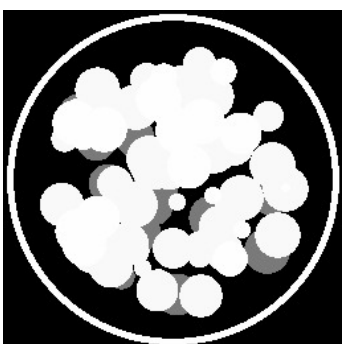

(b)

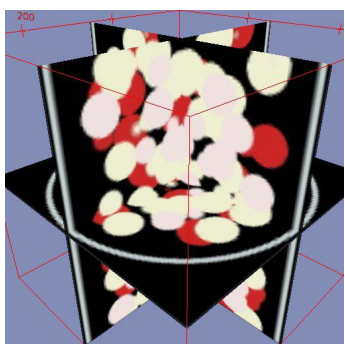

(c)

Figure 1. Simulated object: (a) Central slice of $Z(x)$ volume. (b) Central slice of $\rho(x)$ volume. (c) 3D visualisation of the object imaged into a $512^{3}$ voxel volume.

simulated object. In this study, reconstructed volume size is $N^{3}=256^{3}$ voxels and detector size is $256 \times 192$ pixels. Thus acquisitions have been performed using $512 \times 384$ detector size and downsampled to reconstruct $256^{3}$ volumes.

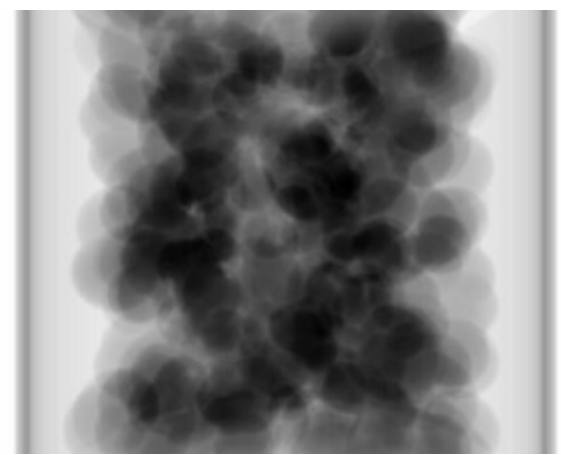

(a)

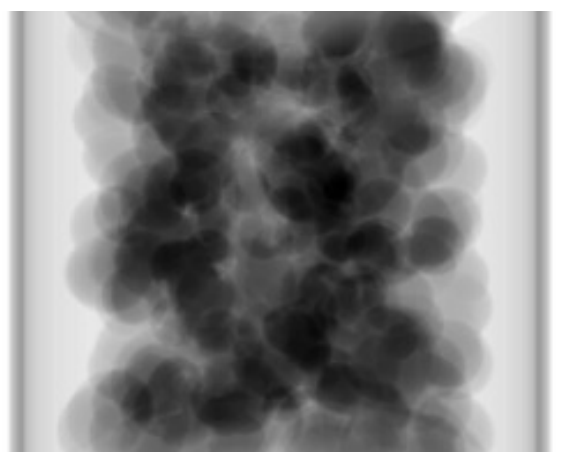

(b)

Figure 2. Projection of synthetic sample at (a) $60 \mathrm{keV}$ and (b) $120 \mathrm{keV}$.

\subsection{Ordered subsets convex reconstruction}

The well-established iterative expectation maximization (EM) algorithm refers to a wide class of tomographic reconstructions providing 3D structural volume of the sample from a set of radiographs. The expectation to observe the measurements, $R$, according to a given sample, $\mu$, denoted $p(R \mid \mu)$ follows a Poisson distribution which can be defined by:

$$
p(R \mid \mu)=\prod_{i} \frac{\mathrm{e}^{-\hat{R}(i)}(\hat{R}(i))^{R(i)}}{R(i) !}
$$

where $i$ is a projection line index and $\hat{R}$, the expected number of counted photons at detector position $i$ is:

$$
\hat{R}(i)=\gamma_{0} \mathrm{e}^{-\sum_{j} w_{i j} \mu(j)},
$$

where $w_{i j}$ is a weight coefficient defining the voxel $j$ contribution on the detector measurement $i . w_{i j}$ is usually proportional to the distance travelled by the line $i$ in the voxel $j$. Based on the likelihood $p(R \mid \mu)$ and the transmission model $\hat{R}$, the method consists of maximizing the $\log \operatorname{likelihood} L=\log p(R \mid \mu)$, ie. finding the solution $\mu$ that minimizes the partial derivative $\partial_{\mu} L$. The solution can be achieved by the Newton-Raphson method iterating in $t:^{7-10}$

$$
\mu_{t+1}=\mu_{t}-\frac{\partial_{\mu_{t}} L}{\partial_{\mu_{t}^{2}}^{2} L}
$$


A penalization $p(\mu)$ is introduced in addition to $p(R \mid \mu)$ to stabilize the solution and avoid divergence during the iterations. $p(\mu)$ is defined with respect to the Gaussian distribution of reconstructed data by: ${ }^{11}$

$$
p(\mu)=e^{-\beta \sum_{j} \sum_{k \in \mathcal{N}(j)} y_{j k} \Phi(\mu(j)-\mu(k))},
$$

where $\beta$ is a relaxation parameter, $\mathcal{N}(j)$ denotes the neighbourhood of pixel $j, \Phi(\cdot)$ is a potential function and $y_{j k}$ a weight factor (usually inverse proportional to the distance between voxels $j$ and $k$ ). In this paper, we use the following LogCosh function as the potential of the regularization: ${ }^{12}$

$$
\Phi(x)=2 g t^{2} \log \cosh \left(\frac{|x|}{t}\right),
$$

where $g$ is a sensitivity parameter. This function attenuates the small variations leading to a better homogeneity of the regions and preserves the edges beyond the given threshold $t$. By replacing $L=\log p(R \mid \mu) p(\mu)$ in Eq. (6), the overall penalized ordered subsets convex (OSC) algorithm consists of iterating in $t$ and subsets $s+1$ in order to update each voxel $j$ of the volume $\mu$ until convergence of the solution:

$$
\begin{aligned}
\mu_{s+1}^{t}(j)= & \mu_{s}^{t}(j)+\alpha^{t} \Delta \mu_{s}^{t}(j), \\
\Delta \mu_{s}^{t}(j)= & \frac{\sum_{i \in S(s)} w_{i j}\left(\hat{R}_{s}^{t}(i)-R(i)\right)-\beta \sum_{k \in \mathcal{N}(j)} y_{j k} \Phi^{\prime}(\mu(j)-\mu(k))}{\sum_{i \in S(s)} w_{i j}\left[\sum_{l} w_{i l}\right] \hat{R}_{s}^{t}(i)+\beta \sum_{k \in \mathcal{N}(j)} y_{j k} \Phi^{\prime \prime}(\mu(j)-\mu(k))}
\end{aligned}
$$

where $\hat{R}_{s}^{t}(i)$ is the expected photon counts computed from $\mu_{s}^{t}$ using Eq. (5), $\alpha^{t}$ is a relaxation parameter, $S(s)$ are the radiographs in the subset $s$ and $\Phi^{\prime}(\cdot)$ and $\Phi^{\prime \prime}(\cdot)$ are respectively the first and second derivative of $\Phi(\cdot)$. The volume obtained by the update using the subset $s$ is used as starting volume of the next subset $s+1$. A main iteration $t$ is completed when all subsets have been processed.

\section{MATERIAL CHARACTERISATION: A PRELIMINARY SIMULATION STUDY}

Let $\mu_{E_{1}}(x)$ and $\mu_{E_{2}}(x)$ be the tomograms reconstructed respectively from two acquisitions at energies $E_{1}$ and $E_{2}$ with the standard OSC algorithm. One can recover the volume pairs $(Z, \rho)$ or $\left(\left[Z^{n} \rho\right], \rho\right)$ by solving one of the following equation systems at each voxel $x$ :

$$
\left\{\begin{array} { l } 
{ \mu _ { E _ { 1 } } ( x ) = A _ { 1 } Z ^ { n } ( x ) \rho ( x ) + B _ { 1 } \rho ( x ) } \\
{ \mu _ { E _ { 2 } } ( x ) = A _ { 2 } Z ^ { n } ( x ) \rho ( x ) + B _ { 2 } \rho ( x ) }
\end{array} \text { or } \left\{\begin{array}{l}
\mu_{E_{1}}(x)=A_{1}\left[Z^{n} \rho\right](x)+B_{1} \rho(x) \\
\mu_{E_{2}}(x)=A_{2}\left[Z^{n} \rho\right](x)+B_{2} \rho(x)
\end{array}\right.\right.
$$

where $A_{1}=\frac{K_{1}}{E_{1}^{k}}, B_{1}=K_{2} f_{K N}\left(E_{1}\right), A_{2}=\frac{K_{1}}{E_{2}^{k}}$ and $B_{2}=K_{2} f_{K N}\left(E_{2}\right)$. Our aim in this section is to define and compare several probabilist material matching functions estimating that a reconstructed voxel corresponds to a material in the material dictionary. Since one can determine $\rho, Z$ or $\left[Z^{n} \rho\right]$ using Eq. 10, a matching function will be based on a comparison of one or several reconstructed material characteristics with their inherent values in the material database.

We consider that reconstructed data $Z(x), \rho(x)$ and $\left[Z^{n} \rho\right](x)$ each follow a Gaussian distribution around an effective material value. Then we can define the probability that a voxel $x$ corresponds to a material $m \in M$ by the following functions:

$$
\left\{\begin{array}{l}
p_{\rho}=p\left(\rho(x)=\rho_{m}\right)=\exp \left(-\left(\rho(x)-\rho_{m}\right)^{2} / \sigma_{\rho_{m}}^{2}\right) \\
p_{Z}=p\left(Z(x)=Z_{m}\right)=\exp \left(-\left(Z(x)-Z_{m}\right)^{2} / \sigma_{Z_{m}}^{2}\right) \\
p_{\left[Z^{n} \rho\right]}=p\left(\left[Z^{n} \rho\right](x)=\left[Z^{n} \rho\right]_{m}\right)=\exp \left(-\left(\left[Z^{n} \rho\right](x)-\left[Z^{n} \rho\right]_{m}\right)^{2} / \sigma_{\left[Z^{n} \rho\right]_{m}}^{2}\right)
\end{array}\right.
$$




\begin{tabular}{|c|c|c|}
\hline$p_{\rho}$ & $p_{Z}$ & $p_{Z^{n}} \rho$ \\
\hline$p\left(\rho(x)=\rho_{m}\right)$ & $p\left(Z(x)=Z_{m}\right)$ & $p\left(\left[Z^{n} \rho\right](x)=\left[Z^{n} \rho\right]_{m}\right)$ \\
\hline$e^{\left(-\frac{\left(\rho(x)-\rho_{m}\right)^{2}}{\sigma_{\rho_{m}}^{2}}\right)}$ & $e^{\left(-\frac{\left(Z(x)-Z_{m}\right)^{2}}{\sigma_{Z_{m}}^{2}}\right)}$ & $e^{\left(-\frac{\left(\left[Z^{n} \rho\right](x)-\left[Z^{n} \rho\right]_{m}\right)^{2}}{\sigma_{\left[Z^{n} \rho\right]_{m}}^{2}}\right)}$ \\
\hline$\sigma_{\rho_{m}}^{2}=\frac{\left(\rho_{m}-\rho_{n}\right)^{2}}{4 \ln \left(p_{t}\right)}$ & $\sigma_{Z_{m}}^{2}=\frac{\left(Z_{m}-Z_{n}\right)^{2}}{4 \ln \left(p_{t}\right)}$ & $\begin{aligned} \sigma_{\left[Z^{n} \rho\right]_{m}}^{2} & =\frac{\left(\left[Z^{n} \rho\right]_{m}-\left[Z^{n} \rho\right]_{n}\right)^{2}}{4 \ln \left(p_{t}\right)} \\
\sigma_{\rho_{m}}^{2} & =\frac{\left(\rho_{m}-\rho_{n}\right)^{2}}{2 \ln \left(p_{t}\right)}\end{aligned}$ \\
\hline $\begin{array}{c}\rho_{n}=\operatorname{argmin}\left\{\rho_{m}-\rho_{m_{i}}\right\} \\
m_{i} \in M \wedge m_{i} \neq m\end{array}$ & $\begin{array}{c}Z_{n}=\operatorname{argmin}\left\{Z_{m}-Z_{m_{i}}\right\} \\
m_{i} \in M \wedge m_{i} \neq m\end{array}$ & $\begin{array}{c}{\left[Z^{n} \rho\right]_{n}=\underset{\operatorname{argmin}}{\operatorname{argmin}}\left\{\left[Z^{n} \rho\right]_{m}-\left[Z^{n} \rho\right]_{m_{i}}\right\}} \\
\rho_{n}=\operatorname{argmin}\left\{\rho_{m}-\rho_{m_{i}}\right\} \\
m_{i} \in M \wedge m_{i} \neq m\end{array}$ \\
\hline
\end{tabular}

Table 2. Singular matching functions investigated according to the reconstructed $\rho(x), Z$ and/or $\left[Z^{n} \rho\right]$ volumes. Complex matching functions (Eq. 12) are obtained by combination of $p_{\rho}$ with $p_{Z}$ or $p_{Z^{n}}$.

or a combination of them such that:

$$
\left\{\begin{array}{l}
p_{Z \rho}=p\left(Z(x)=Z_{m}\right) p\left(\rho(x)=\rho_{m}\right) \\
p_{\left[Z^{n} \rho\right] \rho}=p\left(\left[Z^{n} \rho\right](x)=\left[Z^{n} \rho\right]_{m}\right) p\left(\rho(x)=\rho_{m}\right)
\end{array}\right.
$$

where $\rho_{m}, Z_{m}$ and $\left[Z^{n} \rho\right]_{m}$ are the chemical characteristics of material $m$ in the dictionary $M$ and $\sigma_{\rho_{m}}, \sigma_{Z_{m}}$ and $\sigma_{\left[Z^{n} \rho\right]_{m}}$ are its respective standard deviations. Large deviation will result in a non-accurate estimation since all materials in the dictionary will achieve a good probability (smooth estimate). Conversely, too small deviation will lead to too-discriminant estimates. Let $a_{m}$ be one of the comparison value for material $m$ (i.e. $a_{m}=\left\{\rho_{m}, Z_{m}\right.$ or $\left.\left.\left[Z^{n} \rho\right]_{m}\right\}\right)$. We denote $a_{n}$ the corresponding value for the material $n \in M$ such that $n \neq m$ and $a_{n}=\operatorname{argmin}\left\{a_{m}-a_{m_{i}}, m_{i} \in M \wedge m_{i} \neq m\right\}$. We define the standard deviation $\sigma_{a}$ of $m$ for the material characteristic $a$ by:

$$
\sigma_{a}^{2}=\frac{\left(a_{m}-a_{n}\right)^{2}}{4 \ln \left(p_{t}\right)}
$$

where $p_{t} \in\{0,1\}$ define the distinguishability between $m$ and its closer neighbourhood $n$ in the material dictionary. Setting such a standard deviation for each material allows to locally adjust the difficulty of the probabilist matching function with respect to the chemicals composing the database. A summary of the three single bestmatching functions (Eq. 11) is provided in Tab. 2. Fig. 3 show the probability material distributions of the database Tab. 1 according to the five matching functions (Eq. 11 and Eq. 12) investigated in this paper $\left(p_{t}=0.8\right.$ in this example). Particularly, Fig. 3(f) shows the impact on the distribution when using a restricted material database (containing only the chemicals which are used in our numerical sample) compared to a full database Fig. 3(d), for the same probability function.

In order to quantify the efficiency of each matching function, we first compute numerical acquisitions at two energies using the simulation explained in section 2.1. Then, we reconstruct the volumes $\left(\mu_{1}, \mu_{2}\right)$ using the OSC algorithm detailed in section 2.2. We estimate the volumes $(Z, \rho)$ and $\left(\left[Z^{n} \rho\right], \rho\right)$ by solving Eq. 10 for each voxel. Finally, we apply the classification labelling using each matching function. This classification labels each voxel with the material $m \in M$ such that $m$ maximizes the used probability function. Colored images on Fig. 4(b-f) show the results for each method compared to the reference image (central slice of the simulated object). The number of correct labelling (Corr. in \% on Fig. 4) demonstrates that each function has correctly recovered at least $50 \%$ of the voxels composing the sample from full dictionary. Best result is obtained using $p_{Z \rho}$ even if we can globally denote that differences between different results are not significant. Fig. 5 shows most-likely material results when the material dictionary is restricted to the chemicals composing the acquired sample. The labelling process achieves a better result whatever the matching function. However we can note the high results obtained by $p_{Z}$ and $p_{\left[Z^{n} \rho\right]}$ compared to the others. Thus one can expect to perform an accurate matching from $(Z, \rho)$ volumes as well as than from $\left(\left[Z^{n} \rho\right], \rho\right)$ volumes. 


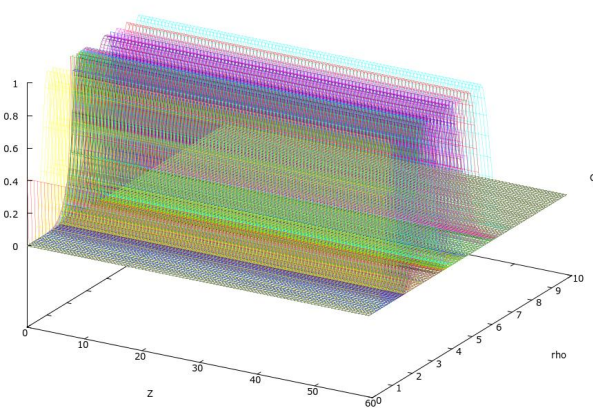

(a) $p_{\rho}$

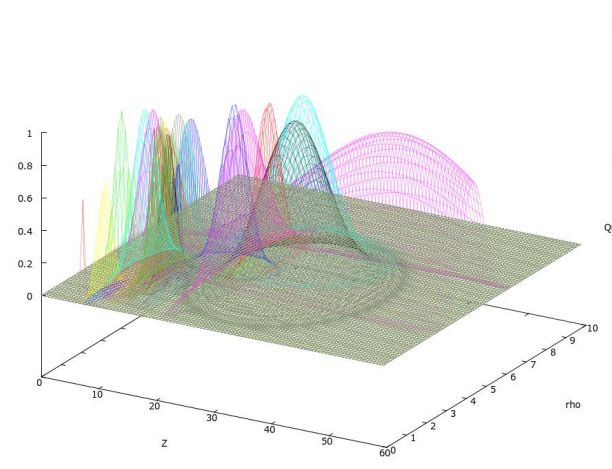

(c) $p_{Z \rho}$

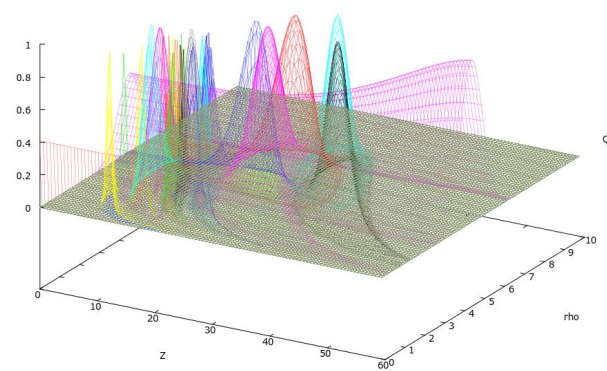

(e) $p_{\left[Z^{n} \rho\right] \rho}$
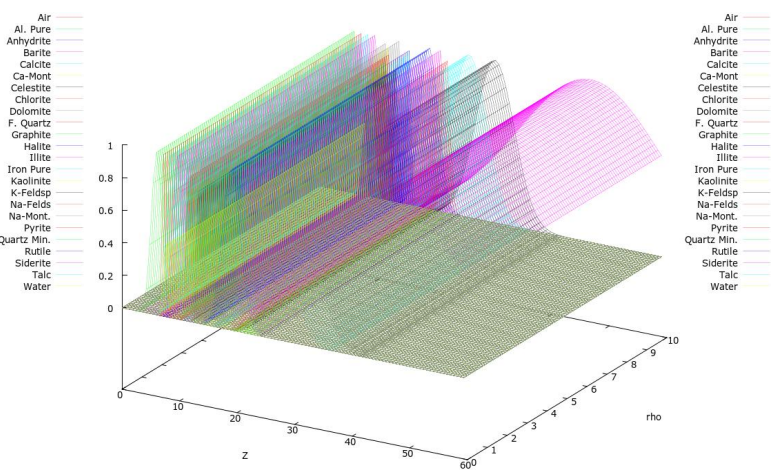

(b) $p_{Z}$
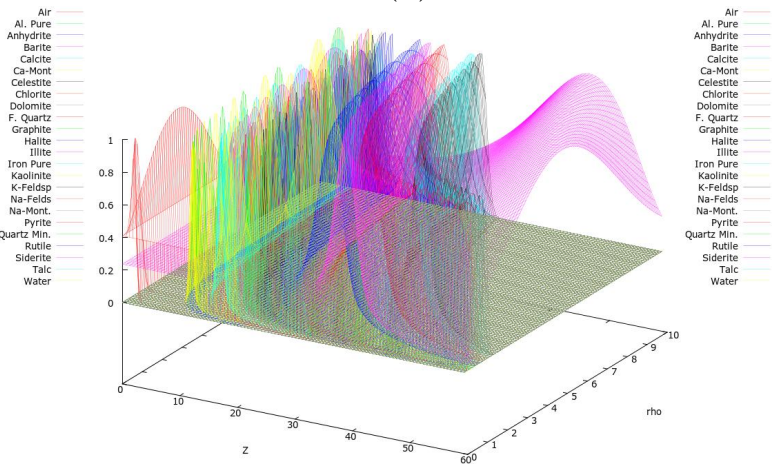

(d) $p_{\left[Z^{n} \rho\right]}$
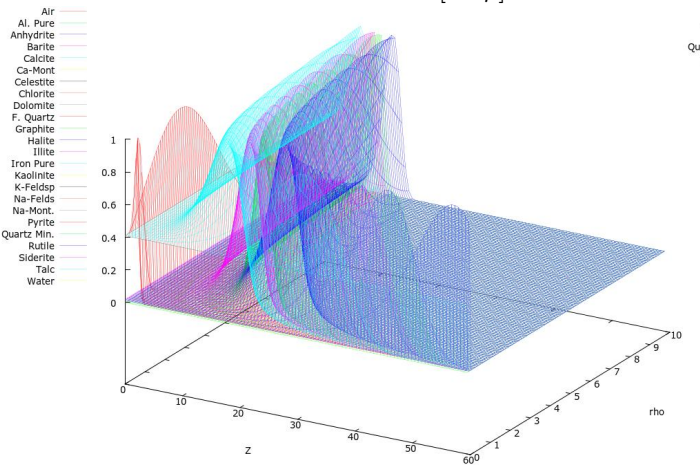

(f) $p_{\left[Z^{n} \rho\right]}$

Figure 3. Material distribution of probabilities according to $Z$ and $\rho$ with respect to the probabilist function: (a) $p_{\rho}$, (b) $p_{Z}$, (c) $p_{Z \rho}$, (d) $p_{\left[Z^{n} \rho\right]}$ and (e) $p_{\left[Z^{n} \rho\right] \rho}$ for the complete material dictionary. (f) $p_{\left[Z^{n} \rho\right]}$ distribution of the material database restricted to the chemicals used in the numerical sample.

\section{DUAL ENERGY RECONSTRUCTION AND MATERIAL MATCHING}

We have observed in section 3 that a material labelling can be performed from $(Z, \rho)$ as well as from $\left(\left[Z^{n} \rho\right], \rho\right)$ volumes. The latter has the advantage to be not limited by the noise in $\rho$ volume. However the matching process developed in the previous section is performed after tomographic reconstruction whereas it could be done during an iterative reconstruction technique, allowing false matching to be corrected during subsequent iterations of the algorithm. Thus we develop now a modified dual-energy OSC algorithm based on the reconstruction of $\left(\left[Z^{n} \rho\right], \rho\right)$ volumes and including a constrained update of the voxels at each iteration. This update enforces each voxel under-reconstruction to be closer to the most likely material in the database.

The forward model of the algorithm is based on the previous estimate of $\left(\left[Z^{n} \rho\right]_{s-1}^{t}, \rho_{s-1}^{t}\right)$. Thus $\mu_{E_{1}}(j)$ and 


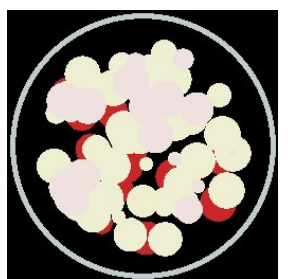

Corr. (in \%)

(a)

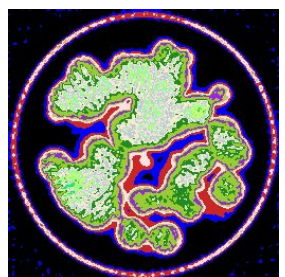

50.23

(b) $p_{\rho}$

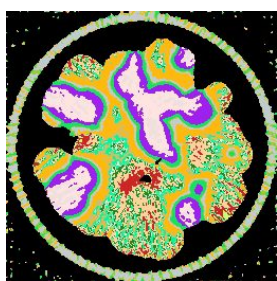

52.08

(c) $p_{Z}$

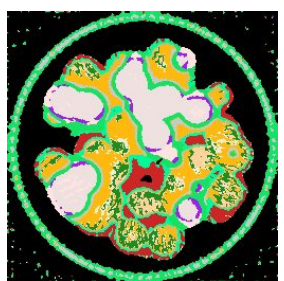

58.28

(d) $p_{Z \rho}$

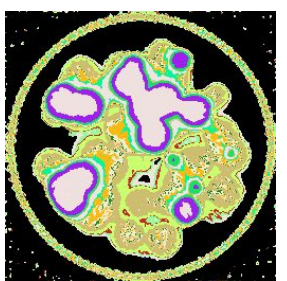

53.87

(e) $p_{\left[Z^{n} \rho\right]}$

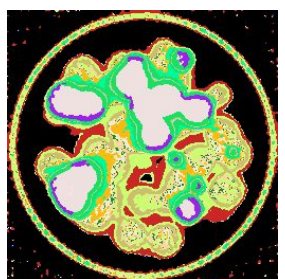

56.21

(f) $p_{\left[Z^{n} \rho\right] \rho}$

Figure 4. Estimate results compared to the original labelling (a). (b) $p_{\rho}$. (c) $p_{Z}$. (d) $p_{Z \rho}$. (e) $p_{\left[Z^{n} \rho\right]}$. (f) $p_{\left[Z^{n} \rho\right] \rho}$, using the full dictionary. Number of correct (Corr.) matching (in \%).

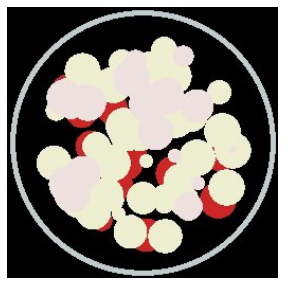

Corr. (in \%)

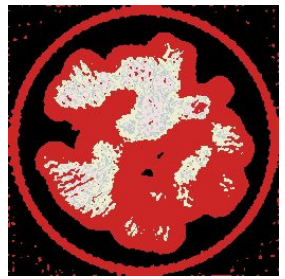

55.04

(b) $p_{\rho}$

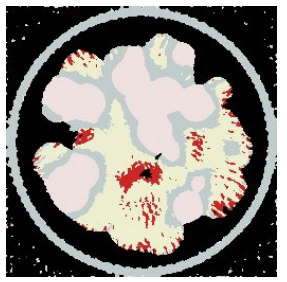

79.04

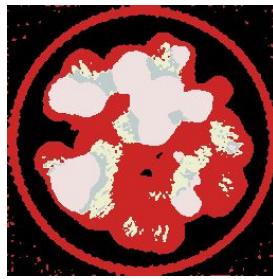

64.48

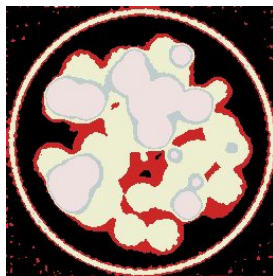

77.18

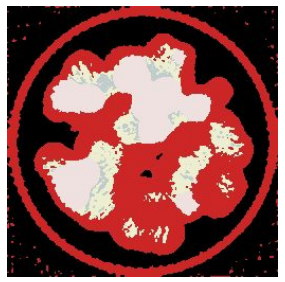

64.68

Figure 5. Estimate results compared to the original labelling (a). (b) $p_{\rho}$. (c) $p_{Z}$. (d) $p_{Z \rho}$. (e) $p_{\left[Z^{n} \rho\right]}$. (f) $p_{\left[Z^{n} \rho\right] \rho}$, using a material dictionary restricted to the chemicals composing the numerical sample. Number of correct (Corr.) matching (in $\%)$.

$\mu_{E_{2}}(j)$ in Eq. 5 are given by:

$$
\left\{\begin{array}{l}
\mu_{E_{1}}(x)=A_{1}\left[Z^{n} \rho\right]_{s-1}^{t}(x)+B_{1} \rho_{s-1}^{t}(x) \\
\mu_{E_{2}}(x)=A_{2}\left[Z^{n} \rho\right]_{s-1}^{t}(x)+B_{2} \rho_{s-1}^{t}(x)
\end{array}\right.
$$

Then we obtain the update values $\Delta \mu_{s}^{t}\left(x, E_{1}\right)$ and $\Delta \mu_{s}^{t}\left(x, E_{2}\right)$ by applying the OSC update step Eq. 9 separately for $E_{1}$ and $E_{2}$. Let $\Delta\left[Z^{n} \rho\right]_{s}^{t}(x)$ and $\Delta \rho_{s}^{t}(x)$ the update values of $\left(\left[Z^{n} \rho\right]_{s-1}^{t}, \rho_{s-1}^{t}\right)$. They are recovered by solving for each $x$ :

$$
\left\{\begin{array}{l}
\Delta \mu_{s}^{t}\left(x, E_{1}\right)=A_{1} \Delta\left[Z^{n} \rho\right]_{s}^{t}(x)+B_{1} \Delta \rho_{s}^{t}(x) \\
\Delta \mu_{s}^{t}\left(x, E_{2}\right)=A_{2} \Delta\left[Z^{n} \rho\right]_{s}^{t}(x)+B_{2} \Delta \rho_{s}^{t}(x)
\end{array}\right.
$$

We add a constrained update at the end of each super-iteration $t$ based on best-matching functions. This update consists of modifying $\left[Z^{n} \rho\right]^{t}(x)$ and $\rho^{t}(x)$ if the most likely material $m$ is such that $p_{\alpha}=p(x=m)>p_{\beta}$, where $p_{\beta}$ is a probability threshold. In such a case, the constrained update performs as follows:

$$
\left\{\begin{array}{l}
{\left[Z^{n} \rho\right]^{t}(x) \leftarrow p_{\alpha}\left[Z^{n} \rho\right]^{t}(x)+\left(1-p_{\alpha}\right)\left[Z^{n} \rho\right]_{m}} \\
\rho^{t}(x) \leftarrow p_{\alpha} \rho^{t}(x)+\left(1-p_{\alpha}\right) \rho_{m}
\end{array}\right.
$$

where $\left[Z^{n} \rho\right]_{m}$ and $\rho_{m}$ are the chemical characteristics of $m$. This constrained update enforces reconstructed data to be closer to the theoretical values of a material $m$ in the dictionary if $m$ is the best likelihood and $p(x=m)$ already provides a high probability (greater than threshold $p_{\alpha}$ ). Matching providing a best estimate lesser than $p_{\alpha}$ are not modified by the constrained update. This constraint has the advantage to make the solution closer to the material in the dictionary.

This algorithm is now applied using the same set of acquisitions at $E_{1}$ and $E_{2}$ than on previous section. We have set in this paper $p_{\beta}=p_{t}=0.8$, and we have used the probability function $p_{\left[Z^{n} \rho\right]}$ in the constrained update since it has provided the average best results in the preliminary study. Central slice of the reconstructed/labelled data are shown on Fig. 6(b-c) using respectively full-database and restricted-database. This technique has correctly labelled more than $66 \%$ (resp. $87 \%$ ) of the voxels containing the sample from the full (resp. restricted) material database. Thus we observe a gain of more than $10 \%$ for the same probability function when using 
the iterative reconstruction with constraint. These results demonstrate the efficiency to combine tomographic reconstruction with material labelling. However result still suffers from material labelling errors, due to the noise and partial volume approximations.

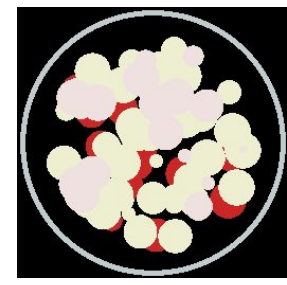

Corr. (in \%)

(a)

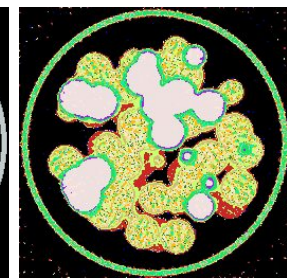

66.41

(b)

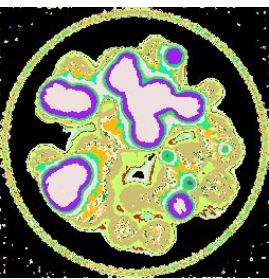

53.87

Fig. 4(e) result.

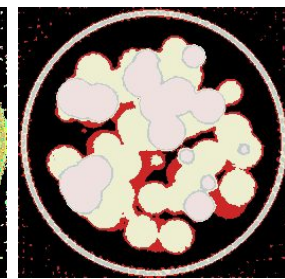

87.91

(c)

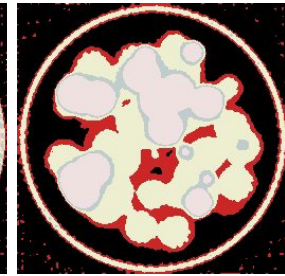

77.18

Fig. 5(e) result.

Figure 6. Estimate results compared to the original labelling (a) using $p_{\left[Z^{n} \rho\right]}$ in the iterative reconstruction: (b) with full material dictionary, (c) using material dictionary restricted to the chemicals composing the sample. Number of correct (Corr.) matching (in \%) among the voxels containing chemicals only. Previous results obtained by sequential processing sequence using the same probability function are repeated for comparison.

\section{CONCLUSION}

In this paper we have investigated several probabilist techniques to label each voxel of a tomogram according to the physical characteristics contained in a material dictionary. These matching techniques are based on a local distinguishability of each material in the database with respect to its closest neighbourhood. Thus one can achieve a material labelling from $(Z, \rho)$ or $\left(\left[Z^{n} \rho\right], \rho\right)$ volumes with equivalent quantitative results. However since it is performed after tomographic reconstruction, the material labelling can not be corrected according to physical models. Since iterative reconstructions are based on such physical properties of X-Ray radiations according to material characteristics, we have proposed a dual-energy OSC algorithm, based on the reconstruction of $\left(\left[Z^{n} \rho\right], \rho\right)$ volumes, including a constrained update at the end of each iteration. This additional update enforces the values of $\left(\left[Z^{n} \rho\right], \rho\right)$ to be closer to the theoretical values of the material $m$ which provides the best probability. Such a process achieves both a better reconstruction of the object and a more accurate labelling compared to a process performing tomographic reconstruction and material classification sequentially. However we have neglected the beam-hardening effects in our simulated study, while it is investigated by our group in another paper. ${ }^{13}$ These techniques can extract $\left(\left[Z^{n} \rho\right], \rho\right)$ projections to reconstruct $\left(\left[Z^{n} \rho\right], \rho\right)$ volumes, avoiding intermediates $\Delta \mu_{s}^{t}\left(x, E_{1}\right)$ and $\Delta \mu_{s}^{t}\left(x, E_{2}\right)$ and correcting for polychromatic spectra at once. A combination of these techniques with the dual reconstruction/labelling proposed in this paper will be investigated in our future works.

\section{REFERENCES}

[1] Alvarez, R. E. and Macovski, A., "Energy-selective reconstructions in x-ray computerised tomography," Physics in medicine and biology 21(5), 733 (1976).

[2] Siddiqui, S. and Khamees, A. A., "Dual-energy ct-scanning applications in rock characterization," in [SPE Annual Technical Conference and Exhibition (Society of Petroleum Engineers, 2004), paper], (2004).

[3] Heismann, B., Leppert, J., and Stierstorfer, K., "Density and atomic number measurements with spectral x-ray attenuation method," Journal of applied physics 94(3), 2073-2079 (2003).

[4] Recur, B., Fauconneau, M., Kingston, A. M., Myers, G., and Sheppard, A. P., "Iterative reconstruction optimisations for high angle cone-beam micro-ct," SPIE Optics and Photonics - accepted at Optical Engineering and Applications. (2014).

[5] Myers, G., "Improving dynamic tomography quality through a maximum a posteriori model," SPIE Optics and Photonics - accepted at Optical Engineering and Applications. (2014).

[6] Nuyts, J., De Man, B., Fessler, J. A., Zbijewski, W., and Beekman, F. J., "Modelling the physics in the iterative reconstruction for transmission computed tomography," Physics in medicine and biology 58(12), R63 (2013). 
[7] Lange, K. and Fessler, J. A., "Globally convergent algorithms for maximum a posteriori transmission tomography," Image Processing, IEEE Transactions on 4(10), 1430-1438 (1995).

[8] De Man, B., Basu, S., Thibault, J.-B., Hsieh, J., Fessier, J., Bouman, C., and Sauer, K., "A study of four minimization approaches for iterative reconstruction in x-ray ct," in [Nuclear Science Symposium Conference Record, 2005 IEEE], 5, 2708-2710, IEEE (2005).

[9] Kamphuis, C. and Beekman, F. J., "Accelerated iterative transmission ct reconstruction using an ordered subsets convex algorithm," Medical Imaging, IEEE Transactions on 17(6), 1101-1105 (1998).

[10] Erdogan, H. and Fessler, J. A., "Ordered subsets algorithms for transmission tomography," Physics in medicine and biology 44(11), 2835 (1999).

[11] Lange, K., "Convergence of em image reconstruction algorithms with gibbs smoothing," Medical Imaging, IEEE Transactions on 9(4), 439-446 (1990).

[12] Blanc-Féraud, L., Charbonnier, P., Aubert, G., and Barlaud, M., "Nonlinear image processing: modeling and fast algorithm for regularization with edge detection," in [Image Processing, 1995. Proceedings., International Conference on], 1, 474-477, IEEE (1995).

[13] Kingston, A., "Title," SPIE Optics and Photonics - accepted at Optical Engineering and Applications. (2014). 